\title{
Candidate genes within known QTL of type I diabetes from mouse models Weikuan $\mathrm{Gu}^{1}$, Ivan Gerling${ }^{2}$, Yan Jiao ${ }^{1}$, Peng $\mathrm{Gao}^{1}$ and Qing Xiong*1,3
}

\author{
Address: ${ }^{1}$ Department of Orthopedic Surgery - Campbell Clinic and Pathology, University of Tennessee Health Science Center, Memphis, TN \\ 38163, USA, ${ }^{2}$ Department of Medicine, University of Tennessee Health Science Center, Memphis, TN 38163, USA and ${ }^{3}$ Department of Computer \\ Science and Technology, Southwest University, P.R. China \\ Email: Qing Xiong* - qxiong1@utmem.edu \\ * Corresponding author
}

from UT-ORNL-KBRIN Bioinformatics Summit 2008

Cadiz, KY, USA. 28-30 March 2008

Published: 8 July 2008

BMC Bioinformatics 2008, 9(Suppl 7):P2 doi:10.1 186/I47/-2105-9-S7-P2

This abstract is available from: http://www.biomedcentral.com/I47I-2105/9/S7/P2

(c) 2008 Gu et al; licensee BioMed Central Ltd.

\section{Introduction}

Quantitative trait loci (QTL) are often positioned within large genomic regions that contain dozens to hundreds of genes. It proves difficult to identify underlying genes by direct experiments. Nevertheless, identification of candidate genes by database mining can be productive by defining the size of the subregion around the optimum QTL map position, coupled with q systematic key word search of genetic and published information. Using a recently developed software package, PGMapper [1], a systematic study has been conducted to examine candidate genes of QTL of type 1 diabetes according to all available literature reports in PubMed and Online Mendelian Inheritance in Man (OMIM).

\section{Materials and methods}

Literature search was conducted with key words "diabetes" and "QTL" in PubMed for every publication up to December 2007. Candidate gene search was conducted with PGMapper [1]. Genes within a QTL region were obtained from the Ensembl database http:// www.ensembl.org/index.html. For every known gene in a QTL, their potential connection with type 1 diabetes was evaluated by searching information from OMIM http:// www.ncbi.nlm.nih.gov/entrez/query.fcgi? $\mathrm{db}=\mathrm{OMIM}$ and PubMed http://www.ncbi.nlm.nih.gov/entrez/ query.fcgi? $\mathrm{db}=$ PubMed.

\section{Results and conclusions}

Our search produced some unexpected results. We examined 9038 genes within the interval of 26 QTL of type 1 diabetes, of which 138 genes were considered to be candidate genes for those QTL. On one hand, there are a large number of candidate genes located in each of those QTL; on the other hand, we also found that some obvious candidate genes of QTL have not yet been investigated. Thus, the comprehensive search of candidate genes for known QTL may yield unexpected benefit for identification of responsive genes underlying diabetes QTL. Our search also indicated that genome resources such as Ensembl database and bioinformatics tools such as PGMapper could be great assistance in searching for candidate genes of QTL.

\section{Acknowledgements}

We wish to acknowledge an NIH, National Institute of Arthritis and Musculoskeletal and Skin Diseases, grant AR5II90 (To WG).

\section{References}

I. Xiong Q, Qiu Y, Gu W: PGMapper: a web-based tool linking phenotype to genes. Bioinformatics 2008, 24(7): $1011-1013$. 